\title{
Modelling Effect of Heart Failure on the Electrical Activity of Sheep Atria
}

\author{
Nouf R Alshwaira ${ }^{1}$, Henggui Zhang ${ }^{1}$ \\ ${ }^{1}$ University of Manchester, Manchester, United Kingdom
}

\begin{abstract}
Heart failure $(H F)$ is associated with cardiac arrhythmias, which impairs cardiac electromechanics that causes dysfunction of cardiac muscle contraction leading to increased risks of morbidity and mortality. Previous studies have revealed that $H F$ causes alteration to the electrophysiological and structural properties of the atria.

The aim of this study was to investigate the primary factor of HF-induced remodelling on the dynamical behaviours of electrical excitation waves in sheep atria.

The biophysically detailed model of sheep atrial action potentials developed by Butters et al was modified to incorporate experimental data of HF-induced remodelling on ion channels. The developed atrial cell models in $H F$ were then incorporated into the $3 D$ anatomical sheep atria model developed in our previous study. The 3D model considered both electrical heterogeneity and tissue anisotropy.

At the cellular level, HF shortened the action potential duration at $90 \%$ of repolarisation $\left(A P D_{90}\right)$. At $3 D$ organ level, activation time of the whole atria was prolonged due to the downregulation of expression of gap junction proteins (Cx43). Consequently, the wavelength of excitation waves was abbreviated, which may help to sustain re-entrant excitation waves in the atria.

This study provides mechanistic insights into the proarrhythmic effect of $\mathrm{HF}$-induced remodeling on ion channels, $\mathrm{Ca}^{2+}$ handling and intercellular coupling in the sheep atria.
\end{abstract}

\section{Introduction}

Atrial fibrillation (AF) is the most common cardiac arrhythmia with features of chaotic and disorganised excitation activity in different regions of the atria, leading to increased mortality rates [1]. Genesis of AF may be attributable to the altered electrical physiological properties of atrial cells, including changes in action potential duration (APD) and effective refractory period (ERP). Since 1959 when Moe et al started studying reentrant circuits associated with AF, several theories been proposed for the mechanisms underlying the initiation and maintenance of AF [2]. For example, ectopic activity in pulmonary veins has been found to be a possible trigger that may initiate AF [3]. Although the chaotic activities were observed in many experimental and computational models of AF, mapping studies demonstrate that AF is mainly driven by organised and high frequency re-entrant circuits [4]. Moreover, the anatomical structure plays an important role in determining the fibrillatory behaviour and dominant frequency of re-entrant activities.

Heart failure $(\mathrm{HF})$ is associated with increased risks of cardiac arrhythmias, including AF. Previous studies have revealed that $\mathrm{HF}$ causes alteration to the electrophysiological and structural properties of the atria. However, the mechanism underlying the proarrhythmic effect of HF is incompletely understood yet.

The aim of this study was to use the biophysical detailed computer model of the sheep atria to investigate the effects of HF-induced remodelling on ion channels at cellular and 3D organ levels with considered electrical heterogeneity and tissue anisotropy. Specifically, the effect of HF-induced remodelling on ion channels in morphology of action potential and its duration at $90 \%$ of repolarisation $\left(A P D_{90}\right)$, AP characteristics and activation time in the 3D model was studied by simulations.

To achieve that, the sheep atria model developed in our previous study was modified to incorporate experimental data of HF-induced remodelling on ion channels and $\mathrm{Ca}^{2+}$ handling. Then, the developed atria cell models of $\mathrm{HF}$ were incorporated into the 3D anatomical model of the sheep atria.

\section{Methods}

\subsection{Single cell simulations}

At the single cell level, the biophysically detailed model of sheep atrial action potentials for different cell types developed by Butters et al [5] including the left atrium (LA), crista terminalis (CT), pectinate muscles (PMs), right atrial appendage (APG), pulmonary veins (PVs) and Bachmann's bundle (BB) was modified to incorporate comprehensive experimental data of HF- 
induced remodeling on ion channels and $\mathrm{Ca}^{2+}$ handling. These ion channels remodelling include downregulation of the L-type calcium current $\left(I_{C a L}\right)$, the slow delayed rectifier potassium current $\left(I_{K S}\right)$, and the transient outward potassium current $\left(I_{t o}\right)$, and upregulation of the inward rectifier potassium current $\left(I_{K 1}\right)$ and the exchanger $\mathrm{Na}^{+}-\mathrm{Ca}^{2+}$ current $\left(I_{\mathrm{NaCa}}\right)$.

The equation governing the time $(t)$ course of cell membrane potential (V) was given by the formulation [6]:

$$
-C_{m} \frac{d V_{m}}{d t}=I_{t o t}+I_{s t i m}
$$

Where, $C_{m}$ is the membrane capacitance, $I_{\text {tot }}$ is the total ionic current and $I_{\text {stim }}$ is the external applied simulation current.

\subsection{D organ level simulation}

The developed atrial cell models with HF were then incorporated into the 3D anatomical sheep atria model in our previous study. The 3D model considered both electrical heterogeneity and tissue anisotropy. The propagation of action potential (AP) in 3D organ level was described using monodomain equation [7]:

$$
\frac{\partial V_{m}}{\partial t}=\nabla \cdot(\mathrm{D} \nabla V)-\frac{I_{i o n}+I_{\text {stim }}}{C_{m}}
$$

Where, $C_{m}$ is the membrane capacitance, $\nabla$ is the gradient operator, $I_{i o n}$ is the total ionic current, $I_{\text {stim }}$ is the external applied simulation current and D is the tensor of diffusion coefficient that describe the AP propagation in relation to the myofibre orientations:

$$
D=D_{\|} a a^{T}+D_{\perp} b b^{T}+D_{\star} c c^{T}
$$

Where, $D_{\|}$is the diffusion coefficient along the fibres, $D_{\perp}$ is the diffusion coefficient orthogonal to the fibres in sheet plan, $D_{\star}$ is the diffusion coefficient orthogonal to fibres and sheet plan, and a, $b$ and $c$ are fibre vectors in the corresponding directions.

\section{Results}

\subsection{Single cell simulation}

Figure 1 shows the simulated AP in control (CTL) and HF conditions by using the LA cell model. It was shown that the AP morphology of the left atrium was triangular and less plateau in phase 2 than in the CTL condition. This is attributable to the HF-induced alteration in some ionic channel currents and $\mathrm{Ca}^{2+}$ handling. The observed changes in AP morphology in simulation were in consistence with experimental measurements [8].

Further analysis showed that, $A P D_{90}$ in the LA was significantly shortened by $22.74 \%$ in HF condition as compared to CTL. Moreover, the AP characteristics were altered, resulting in a decrease in the overshoot (OS) by $10.32 \%$, a slight reduction of the action potential amplitude (APA) by $1.01 \%$. The resting potential was more hyperpolarised from $-76.51 \mathrm{mV}$ (CTL) to -77.63 $\mathrm{mV}$ (HF). The maximum upstroke velocity $d V / d t_{\text {MAX }}$ was decreased by $1.98 \%$ (Figure 1 ).

The action potential duration restitution curve (APDR) that describe the rate-dependence of the $A P D_{90}$ was flattened by $\mathrm{HF}$ as compared to CTL condition. The computed maximum slope of the $A P D R_{50}$ and $A P D R_{90}$ were decreased by $40.84 \%$ and $24.24 \%$, respectively in the HF condition (Figure 2).

For more detailed analysis, the inclusive and exclusive methods [9] were used to investigate the relative contribution of $I_{C a L}$ and $I_{K 1}$ currents in the predominant effect of APD shortening in sheep atrium (Figure 3).

\subsection{D organ level simulation}

Figure 4 shows simulated excitation pattern in the 3D anatomical model of the sheep atria that considered the electrical heterogeneity and tissue anisotropy in both CTL and HF conditions.

In CTL condition the total activation time (the time when each point reach the maximum upstroke velocity) of the propagation of the electrical wave from the sinus node (SA) to the whole atria was $93 \mathrm{~ms}$. Although there was a reduction of the conduction velocity in HF condition (data not shown here), the activation time was less than that in CTL condition $(63 \mathrm{~ms})$. However, in the 3D simulation in HF condition with a consideration of decreased gap junctional coupling $(\mathrm{Cx} 43)$ by $30 \%$, the total activation time was increased to $174 \mathrm{~ms}$. With a consideration of reduction of $\mathrm{Cx} 43$ by $50 \%$, the total activation time became $169 \mathrm{~ms}$, but with regions being not activated in the LA and the Right atrium (RA). These results highlighted the importance of gap junction remodelling in impairing atrial excitation (Figure 4), supporting the hypothesis the deficiency of gap junctions in cardiac myocytes may contribute to arrthymogenesis with unhealthy human heart [10].

A
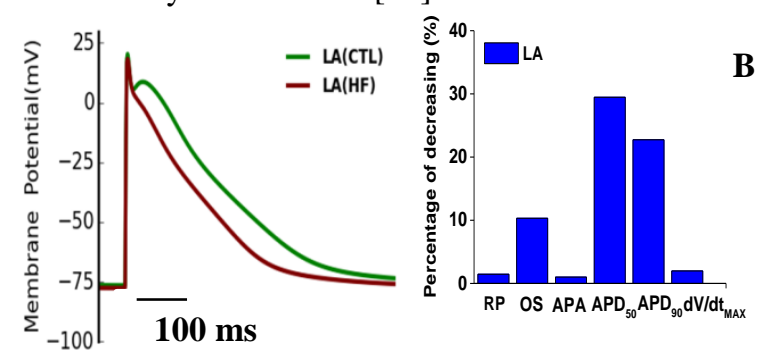

Figure 1. Time course of action potential (AP) of the left atria (LA) in CTL (green) and HF (red) conditions with $\mathrm{BCL}=1000 \mathrm{~ms}(\mathrm{~A})$. Percentage of decrease in AP characteristics of the LA in CTL and HF conditions 
includes: resting potential (RP), overshoot (OS), action potential amplitude (APA), $A P D_{50}$ and $A P D_{90}$, respectively, and maximum upstroke velocity $d V / d t_{\text {MAX }}$.

Ai

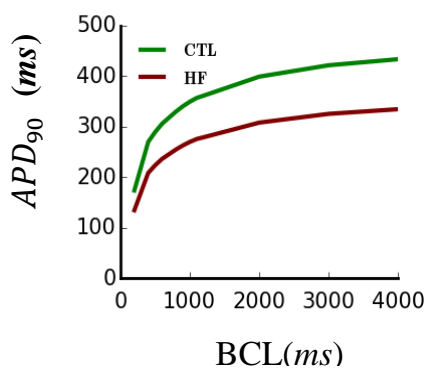

B

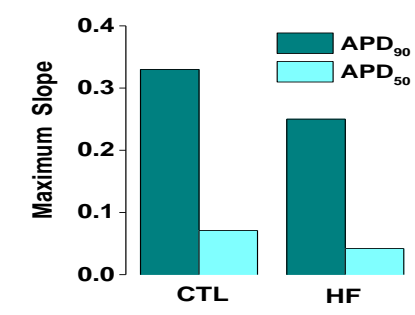

Figure 2. Action potential duration at $90 \%$ and $50 \%$ of repolarisation and their restitution curves $\left(A P D R_{90}\right)$ and $\left(A P D R_{50}\right)$ in CTL (green) and HF conditions (red) (Ai, Aii). Maximum slope of $A P D R_{90}$ and $A P D R_{50}$ (B) restitution curves.
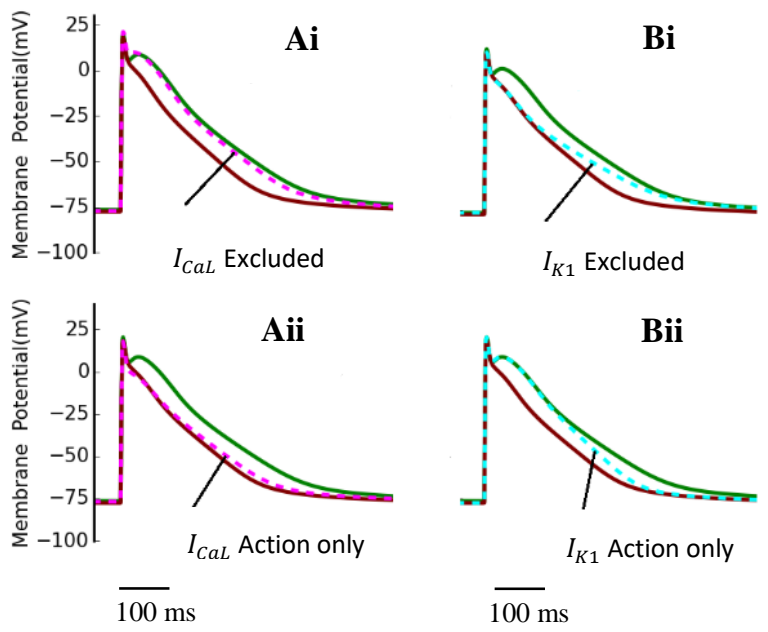

Figure 3. The role of individual remodelled of ionic channels in HF condition. Action potential (AP) was computed in CTL (green), and HF-induced remodelling with all changes in ionic current considered $\left(I_{C a L}, I_{K s}\right.$, $I_{t o}, I_{K 1}$ and $I_{N a C a}$ ) (red). HF remodelling of $I_{C a L}$ was omitted, and HF action of $I_{C a L}$ was only considered (Ai, Aii, pink). HF remodelling of $I_{K 1}$ was omitted, and HF action of $I_{K 1}$ was only considered (Bi, Bii, cyan). BCL $=1000 \mathrm{~ms}$

\section{CTL}

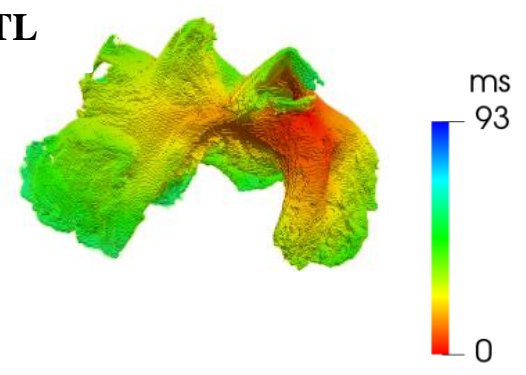

HF

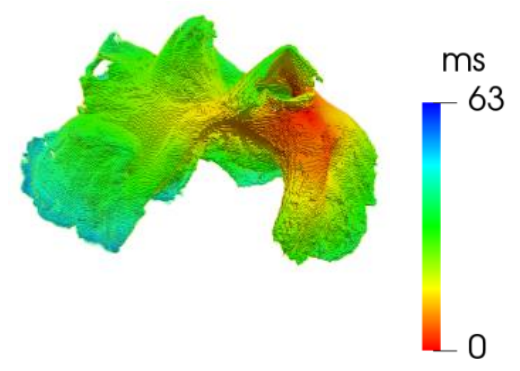

HF (Cx43 reduction by 30\%)

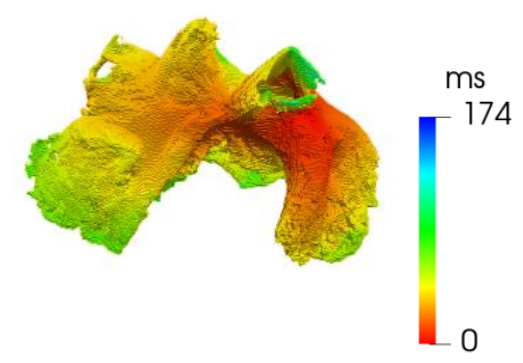

\section{HF ( $\mathrm{Cx} 43$ reduction by $50 \%)$}

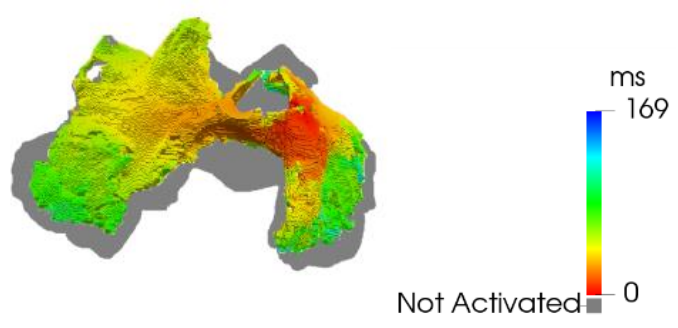

Figure 4. Anterior view of the activation time sequence on the whole sheep atria under control condition (CTL), heart failure (HF), HF condition with downregulation of Connexin (Cx43) expression by $30 \%$, and $50 \%$. The colour bar is an indication of running time from red to blue. Gray colour indicates inactivated region. 


\section{Discussion and conclusion}

In this study, we modified sheep atria model from our pervious study to incorporate experimental measurements of HF-induced remodeling of ion channels, intracellular $\mathrm{Ca}^{2+}$ handling and intercellular coupling for investigating the consequences of heart failure on action potential characteristics at cellular level and activation time at the organ level. These findings were consistent with experimental results. At cellular level, Our simulation results showed that the downregulation of $I_{C a L}, I_{K s}, I_{t o}$ currents and the upregulation of the $I_{K 1}$ and $I_{\mathrm{NaCa}}$ currents caused significant shortening of the action potential duration APD. This is consistent with findings from Clarke et al [8]. To identify the primary factor that produce the reduction of $A P D_{90}$, we applied the inclusive and exclusive methods [9] for more detailed analysis. It has been found that the downregulation of $I_{C a L}$ has the most influence on the shortening of APD in sheep atrial myocytes as shown in figure 3 . Furthermore, the AP restitution curve in $\mathrm{HF}$ condition was flattened. It was shown APD was shortened across all BCLs in HF condition compared to CTL condition, with least rate adaptation in HF condition as there was a decrease in the maximal slope of APDR in HF condition. The loss of rate adaptation of atrial excitation at the cellular level may facilitate high frequency re-entrant circuits at 3D simulation results [11].

Our 3D simulation results showed that the activation time in HF condition was decreased despite of the reduction of the conduction velocity (data are not shown here). However, HF-induced remodelling with a downregulation $\mathrm{Cx} 43$ expression by $30 \%$, activation time was prolonged by $176 \%$. Furthermore, with a downregulation of $\mathrm{Cx} 43$ expression by $50 \%$, there was a $168 \%$ increase in the activation time, but with inactivated regions in the LA and the RA. These results emphasis the changes in gap junctions may contribute to the development pro-arrhythmic effects in diseased heart.

In conclusion, an investigation into the effects of HFinduced ionic channel remodelling and $\mathrm{Ca}^{2+}$ handling on electrophysiological function of sheep atrial cells and tissue has revealed shortened atrial APD and prolonged activation time as potential mechanisms for the proarrhythmic of HF in the sheep atria.

\section{Acknowledgements}

This project was funded by Imam Abdulrahman Bin Faisal University, Dammam, Kingdom of Saudi Arabia.

\section{References}

[1] P. A. Wolf, J. B. Mitchell, C. S. Baker, W. B. Kannel, and R. B. D'Agostino, 'Impact of Atrial
Fibrillation on Mortality, Stroke, and Medical Costs', Arch. Intern. Med., vol. 158, no. 3, p. 229, Feb. 1998.

[2] J. W. Waks, Clinical Fellow in Cardiac Electrophysiology, Harvard Medical School, Harvard-Thorndike Electrophysiology Institute and Arrhythmia Service, Beth Israel Deaconess Medical Center, Boston, US, M. E. Josephson, and Herman C. Dana Professor of Medicine, Harvard Medical School, Chief of the Cardiovascular Division, Beth Israel Deaconess Medical Center and Director, Harvard-Thorndike Electrophysiology Institute and Arrhythmia Service, Beth Israel Deaconess Medical Cent, 'Mechanisms of Atrial Fibrillation - Reentry, Rotors and Reality', Arrhythmia Electrophysiol. Rev., vol. 3, no. 2, p. 90, 2014.

[3] M. Haïssaguerre et al., 'Spontaneous Initiation of Atrial Fibrillation by Ectopic Beats Originating in the Pulmonary Veins', N. Engl. J. Med., vol. 339, no. 10, pp. 659-666, Sep. 1998.

[4] M. Vaquero, D. Calvo, and J. Jalife, 'Cardiac fibrillation: From ion channels to rotors in the human heart', Heart Rhythm, vol. 5, no. 6, pp. 872-879, Jun. 2008.

[5] T. D. Butters, O. V. Aslanidi, J. Zhao, B. Smaill, and H. Zhang, 'A novel computational sheep atria model for the study of atrial fibrillation', Interface Focus, vol. 3, no. 2, pp. 20120067-20120067, Feb. 2013.

[6] A. L. Hodgkin and A. F. Huxley, 'A quantitative description of membrane current and its application to conduction and excitation in nerve', J. Physiol., vol. 117, no. 4, pp. 500-544, Aug. 1952.

[7] R. H. Clayton and A. V. Panfilov, 'A guide to modelling cardiac electrical activity in anatomically detailed ventricles', Prog. Biophys. Mol. Biol., vol. 96, no. 1-3, pp. 19-43, Jan. 2008.

[8] J. D. Clarke et al., 'Perturbed atrial calcium handling in an ovine model of heart failure: Potential roles for reductions in the L-type calcium current', J. Mol. Cell. Cardiol., vol. 79, pp. 169-179, Feb. 2015.

[9] H. Zhang, C. Garratt, J. Zhu, and A. Holden, 'Role of up-regulation of in action potential shortening associated with atrial fibrillation in humans', Cardiovasc. Res., vol. 66, no. 3, pp. 493-502, Jun. 2005.

[10] J. H. Smith, C. R. Green, N. S. Peters, and N. J. Severst, 'Altered Patterns of Gap Junction Distribution in Ischemic Heart Disease', vol. 139, no. 4, p. 21, 1991.

[11] M. Allessie, 'Electrical, contractile and structural remodeling during atrial fibrillation', Cardiovasc. Res., vol. 54, no. 2, pp. 230-246, May 2002.

Address for correspondence.

Nouf R Alshwaira. Room 3.06 Schuster Building, School of Physics and Astronomy.The university of Manchester, Oxford Road, M13 9PL.nouf.alshwaira@postgrad.manchester.ac.uk 